\title{
Synthesis of antibacterial silver-based nanodisks and dendritic structures mediated by royal jelly $\dagger$
}

Cite this: RSC Adv., 2014, 4, 1650

\author{
Raquel Mendoza-Reséndez, ${ }^{* a}$ Alberto Gómez-Treviño, ${ }^{\mathrm{b}}$ Enrique Díaz Barriga- \\ Castro, ${ }^{c}$ Nuria O. Núñez ${ }^{d}$ and Carlos Luna*c
}

\begin{abstract}
The one-step preparation of silver nanoparticles and dendritic structures mediated by aqueous royal jelly solutions has been investigated for the first time. It has been found that royal jelly (RJ) is a complex organic matrix that can be simultaneously used as a reducing and stabilizing agent in the chemical synthesis of colloidal silver-based nanostructures from aqueous $\mathrm{AgNO}_{3}$ solutions, without the requirement of additional reagents or heating sources to initiate the oxidation-reduction reactions. The resulting product consisted of very fine single-crystal disks of $\mathrm{Ag}$ and silver 4,4'dimethyldiazoaminobenzene (a triazenic compound). Both kinds of particles tended to coalesce and form supramolecular dendritic structures, the $\mathrm{AgNO}_{3} / \mathrm{RJ}$ weight ratio chosen in the synthesis being a key parameter to control the crystal growth and the microstructural properties of the Ag nanodisks. Data obtained from Fourier transform infrared and Raman spectroscopy analysis indicated that these nanostructures are coated by RJ biomolecules (residues of proteins and carbohydrates). In vitro biological assays showed that these nanostructures exhibit a promising enhanced antibacterial activity against both Gram-positive and Gram-negative bacteria.
\end{abstract}

Received 9th October 2013

Accepted 19th November 2013

DOI: $10.1039 / c 3 r a 45680 c$

www.rsc.org/advances result of such research efforts, there currently is a large variety of synthetic routes available that yield to the successful preparation of $\mathrm{Ag}$ particles with nanometric sizes. However, these methods invariably involve the use of reagents that are hazardous to human health and have a serious impact on the environment. Therefore, the design of more sustainable approaches for the synthesis of Ag nanostructures is pressingly needed.

Recently, several investigations have shown that the reducing and stabilizing agents used in the conventional production of nanoparticles via oxidation-reduction reactions, which usually have an important environmental impact, can be replaced with extracts or aqueous solutions of natural products (leaves, seeds, fruits, roots, and honey, among others) due to their high antioxidant potential. ${ }^{6-10}$ These alternative synthetic routes offer interesting advantages over the conventional synthesis: they are quite simple, economically competitive and reduce risks to human health and the environment. ${ }^{11}$ In addition, these more sustainable procedures provide new approaches for the stabilization and functionalization of nanostructures especially interesting for applications in biomedicine including drug delivery and tissue imaging. ${ }^{11,12}$ Among the more relevant contributions in this interesting topic, remarkable advances have been made with the use of aqueous honey solutions in the synthesis of various nanomaterials. In this fashion, Philip has prepared $4 \mathrm{~nm}$ silver nanoparticles ${ }^{7}$ and $15 \mathrm{~nm}$ gold nanoparticles in presence of aqueous honey solutions. ${ }^{8}$ Most recently, Madhusudana Reddy et al. have reported the synthesis of anisotropic palladium nanoparticles with sizes 
of 5 to $40 \mathrm{~nm}$ (ref. 13) and Venu et al. ${ }^{14}$ have synthesized ultrafine Pt nanoparticles and nanowires through similar synthetic procedures. The power antioxidant of honey has been ascribed to its high content of mono- and disaccharides (mainly fructose, glucose and sucrose $)^{13,15,16}$ and proteins, ${ }^{17-20}$ and the stabilizing function of honey has been mainly associated to its proteins. ${ }^{7}$

It is well known that royal jelly (RJ) is rich in proteins, monosaccharides, polyphenols and vitamins that provide it a potent antioxidant activity. ${ }^{21}$ Therefore, RJ solutions should serve as very suitable reaction medium in green syntheses of nanomaterials. However, it is worth noting that, even though the composition of royal jelly has been studied since the early work of von Planta published in $1888,{ }^{22,23}$ the complete identification of the complex composition of royal jelly is a research issue that remains quite open, and the development of new characterization techniques is allowing the discovery of new compounds. For instance, 19 new proteins have been recently identified in RJ by one-dimensional gel electrophoresis and gelfree proteomic approaches. ${ }^{24}$ In addition, it has been found that RJ contains several carboxylic acids, being the only known natural source of 10-hydroxy-trans-2-decenoic acid, which is named "royal jelly acid" ${ }^{5}$ and that have potent antibiotic properties. $^{26}$

Encouraged by these promising results and the unique antioxidant and bioactivity properties of royal jelly (RJ), we have investigated in the present contribution for the first time the potential of water solutions of royal jelly as reducing and stabilizing agent in the eco-friendly synthesis of Ag-based nanostructures and their antibacterial activity.

\section{Experimental techniques}

\section{Chemical reagents}

Raw royal jelly was obtained from a local beekeeper in northeastern Mexico. Silver nitrate $\left(\mathrm{AgNO}_{3}, 99+\%\right)$ was purchased from Sigma-Aldrich and was used as received. The water added in all experiments was doubly distilled.

\section{Synthesis of samples}

Several experiments were carried out in triplicates using different $\mathrm{AgNO}_{3} / \mathrm{RJ}$ weight ratios. Table 1 summarizes the synthetic conditions of the most representative experiments. In these experiments, an aqueous royal jelly solution was prepared mixing raw royal jelly with bi-distilled water by vigorous stirring

Table 1 Relationship between the concentration between the silver nitrate and royal jelly used in the synthesis of based-silver samples. All experiments were carried out under vigorous stirring and at room temperature

\begin{tabular}{lllc}
\hline Sample & $\begin{array}{l}\mathrm{AgNO}_{3} \\
{[\mathrm{M}]}\end{array}$ & $\begin{array}{l}\text { Royal jelly } \\
{\left[\mathrm{g} \mathrm{m} \mathrm{m}^{-1} \mathrm{H}_{2} \mathrm{O}\right]}\end{array}$ & $\begin{array}{l}\mathrm{AgNO}_{3} / \mathrm{RJ} \\
\mathrm{wt} \%\end{array}$ \\
\hline $\mathrm{S}-0.4 \%$ & 0.005 & 0.2 & 0.42 \\
$\mathrm{~S}-42 \%$ & 0.5 & 0.2 & 42.47 \\
$\mathrm{~S}-85 \%$ & 0.5 & 0.1 & 84.94 \\
$\mathrm{~S}-0.4 \% \mathrm{~S}$ & 0.005 & 0.2 & 0.42
\end{tabular}

and keeping the concentrations $\mathrm{RJ} /$ water of $0.1 \mathrm{~g} \mathrm{ml}^{-1}$ or $0.2 \mathrm{~g}$ $\mathrm{ml}^{-1}$. Afterwards, $10 \mathrm{ml}$ of the aqueous RJ solution was mixed under vigorous stirring on a magnetic stir plate and at room temperature, with $10 \mathrm{ml}$ of $0.5 \mathrm{M}$ or $0.005 \mathrm{M}$ aqueous solution of silver nitrate (Table 1). Then, the resulting solution was kept under moderate stirring for 24 hours. During the first hours of synthesis, the color of the solution changed from initial light yellow to darker yellow-brown, indicating the reduction of the $\mathrm{Ag}^{+}$ions. After $24 \mathrm{~h}$, the resulting products were purified by successive centrifugation and redispersed in distilled water. Finally, they were named according to the $\mathrm{AgNO}_{3} / \mathrm{RJ}$ weight ratio chosen in their synthesis (samples $\mathrm{S}-0.4 \%, \mathrm{~S}-42 \%$ and S-85\%, see Table 1).

It is pertinent to remark that it was not necessary to regulate the $\mathrm{pH}$ of the reaction media using additional acidic or basic compounds. The $\mathrm{pH}$ is a determining synthetic parameter that affects the reaction kinetics, and therefore, also affects to the average particle size of biosynthesized nanoparticles. For instance, Philip $^{7}$ recently showed that the average particle diameter of $\mathrm{Ag}$ nanoparticles synthesized using aqueous honey solutions, decreases from around 15 down to $4 \mathrm{~nm}$ as the $\mathrm{pH}$ values was increased from 6.5 to 8.5 , increasing also the particle uniformity. In our experiments, the $\mathrm{pH}$ values of the reaction solutions before mixed were 4.0 and 6.3 for $\mathrm{RJ}$ and $\mathrm{AgNO}_{3}$ solutions, respectively. The $\mathrm{pH}$ values of the reaction media were close to $4.0,6.5$ and 7.5 for samples $\mathrm{S}-0.4 \%, \mathrm{~S}-42 \%$ and $\mathrm{S}-85 \%$ respectively, and these values remained unchanged during the reactions.

In an additional experiment, sample $\mathrm{S}-0.4 \%$ was diluted in $50 \mathrm{ml}$ of distilled water and heated up to reach the boiling temperature under vigorously stirring until the sample was completely dried. The obtained powder was named S-0.4\%S (Table 1).

\section{Characterization techniques}

Powder X-ray diffraction (XRD) measurements were carried out using a X'pert Pro X-ray diffractometer (PANalytical) with $\mathrm{Cu} \mathrm{K} \alpha$ $(\lambda=1.5418 \AA)$ radiation. The mean coherence length, $L$, associated to the more intense diffraction peaks were estimated from the full width at half maximum (FWHM) of the corresponding diffraction peaks using the Scherrer equation: ${ }^{27}$

$$
L=\frac{0.9 \lambda}{\beta \cos \theta}
$$

where $\lambda$ is the $\mathrm{X}$-ray wavelength, $\beta$ is the broadening of the diffraction peak and $\theta$ is the Bragg angle. The morphology and microstructure of the samples were examined by conventional and high resolution transmission electron microscopy (TEM and HRTEM) and selected area electron diffraction (SAED) using a FEI-TITAN $80-300 \mathrm{kV}$ microscope operated at an accelerating voltage of $300 \mathrm{kV}$. The specimen for these studies was prepared by depositing and evaporating a drop of the colloidal solution onto lacey carbon coated copper grids. The elements present in the samples were determined using an energy dispersive spectrometry analyzer (EDS) integrated in the transmission electron microscope. HRTEM images were 
analyzed by fast Fourier transform (FFT) using the software Digital Micrograph (Gatan software, Inc). These images and the SAED patterns were interpreted with patterns simulated using the "Web-based Electron Microscopy Application Software: Web-EMAPS". ${ }^{28}$ Fourier transform infrared (FTIR) and Raman spectroscopic measurements were carried out to gain information about the binding between the Ag-based nanostructures and bio-molecules of RJ. The FTIR analyses were performed using a Nicolet 510 Fourier transform spectrometer with the samples diluted in $\mathrm{KBr}$ pellets. Raman spectra were collected in a Horiba HR800 UV Confocal Raman Microscope. For these measurements a green laser $(532.14 \mathrm{~nm})$, working at 600 line per $\mathrm{mm}, 100 \times$ objective, $20 \mathrm{~mW}$, and $0.1 \mathrm{~mm}$ pinhole, was used. The ultraviolet-visible spectra of the nanostructured samples and the aqueous royal jelly were recorded using a Thermo scientific Evolution 60S UV-Vis spectrophotometer and distilled water dispersive medium of samples.

\section{Antibacterial assays}

The antibacterial activity of samples was proved against Salmonella typhimurium (ATCC 14028) and Staphylococcus aureus (ATCC 25923). Radial diffusion assays (RDA) were realized using a bacteria density of 0.6 O.D. units for both strains. Assays were carried out separately in $100 \times 15 \mathrm{~mm}$ Petri dishes poured with $20 \mathrm{ml}$ nutrient agar media and inoculated with each one of the bacterial strains. A $20 \mu$ l aliquot of RJ aqueous solution $(1: 10 \mathrm{v} / \mathrm{v})$ or $20 \mu \mathrm{l}$ of aqueous colloidal suspensions of the biosynthesized nanoparticles with a concentration of $26 \mathrm{mg}$ $\mathrm{ml}^{-1}$ were dropped in the middle of each dish. All preparations were incubated for $24 \mathrm{~h}$ at $37^{\circ} \mathrm{C}$. Once incubation time elapsed, growing inhibition areas were measured to establish the antibacterial effect of the biosynthesized nanoparticles.

Bacterial strains were tested in a Minimal Inhibitory Concentration (MIC) assay to determine the lowest concentration obtained by serial dilutions showing to inhibit visible growth of a microorganism after incubation. Cultures were prepared in TSB media in 96 well plates and immediately after preparation they were incubated at $37{ }^{\circ} \mathrm{C}$ during 24 hours. Samples were tested against both bacterial strains from $1: 200$ to $1: 0.15$. Once incubation time elapsed, the absorbance of the wells was read at $540 \mathrm{~nm}$. The minimal concentration for growth inhibition was estimated by reference to each strain positive control.

\section{Results and discussion}

\section{Microstructural and morphological studies}

Fig. 1a displays typical XRD patterns of silver-based samples synthesized using aqueous royal jelly solutions. The peaks found around $2 \theta$ value of $38.1^{\circ}$ are ascribed to the (111) planes of face-centered cubic (fcc) metallic silver (JCPDS card no. 040783 ), whereas the peaks centered at $2 \theta$ values around $26.45^{\circ}$, $27.80^{\circ}, 32.18^{\circ}, 46.26^{\circ}, 54.85^{\circ}$ and $57.50^{\circ}$ indicate the presence of a crystalline phase of silver $4,4^{\prime}$-dimethyldiazoaminobenzene $\left(\mathrm{C}_{14} \mathrm{H}_{14} \mathrm{AgN}_{3}\right.$, JCPDS card. 35-1540), which is a triazene that very recently have been found in form of single-crystal nanodisks in
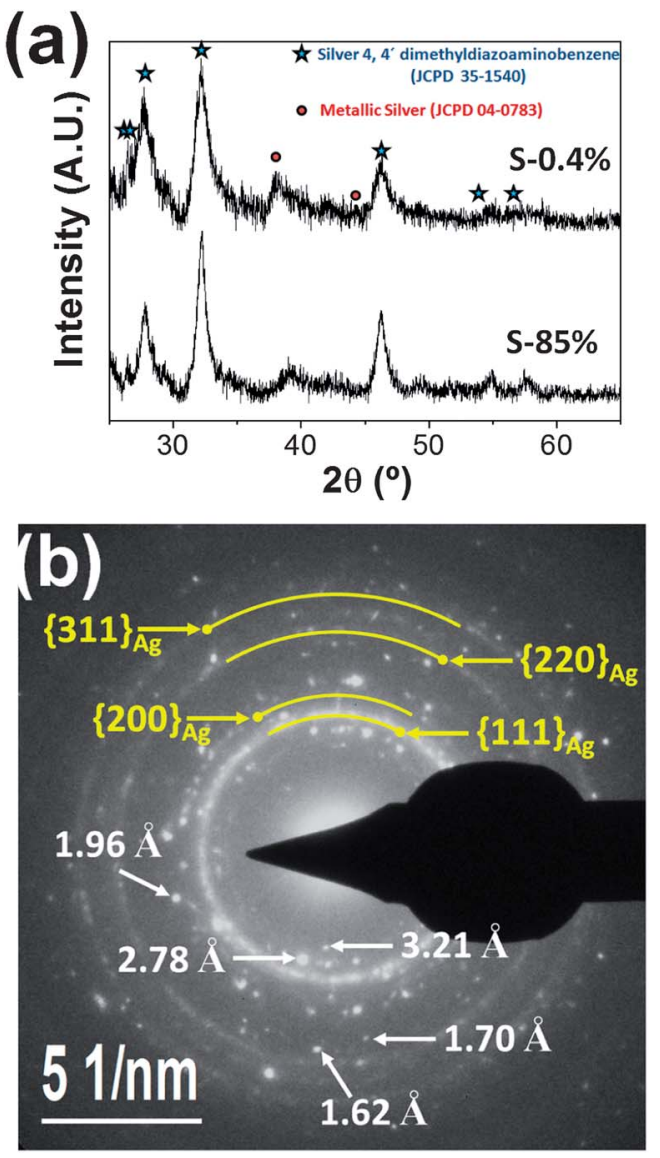

Fig. 1 (a) XRD patterns of samples S- $0.4 \%$ and S- $85 \%$. (b) SAED pattern of an assembly of nanoparticles of sample S- $42 \%$.

the synthesis of silver nanostructures mediated by extracts of Capsicum annuum var. aviculare. ${ }^{29}$

The peak broadness of all diffraction peaks observed in Fig. 1a indicates that both crystalline phases have a nanostructured character. The mean coherence lengths $L$ of samples S- $0.4 \%$ and S-85\% estimated from the peaks more intense of the two phases and using the Scherrer equation were 7 and $12 \mathrm{~nm}$ for the organometallic compound, and 4 and $3 \mathrm{~nm}$ for the metallic silver, respectively.

The presence of both crystalline phases was also evidenced in all samples by SAED analysis. As an illustrative example, Fig. 1b shows a typical SAED pattern of sample S-42\%. It is characterized by several spotty rings that can be associated to the (111), (200), (220) and (311) planes of the fcc metallic silver. Moreover, spots corresponding to lattice spacing values of 3.21, $2.78,1.96,1.70$ and $1.62 \AA$, which correspond to the silver $4,4^{\prime}$ dimethyldiazoaminobenzene crystals, were clearly observed.

Fig. 2a shows a TEM micrograph of the sample S-0.4\%. This image reveals that this sample is constituted by very fine welldispersed particles with a mean diameter of $7 \pm 3 \mathrm{~nm}$ (see their size distribution in Fig. 2b). Comprehensive studies by HRTEM showed that the majority of these particles are Ag single crystals viewed along the [110] direction and with diameters around $4 \mathrm{~nm}$ (the inset of Fig. 2a shows a typical HRTEM of these nanoparticles). It suggests that these fine particles are Ag single- 

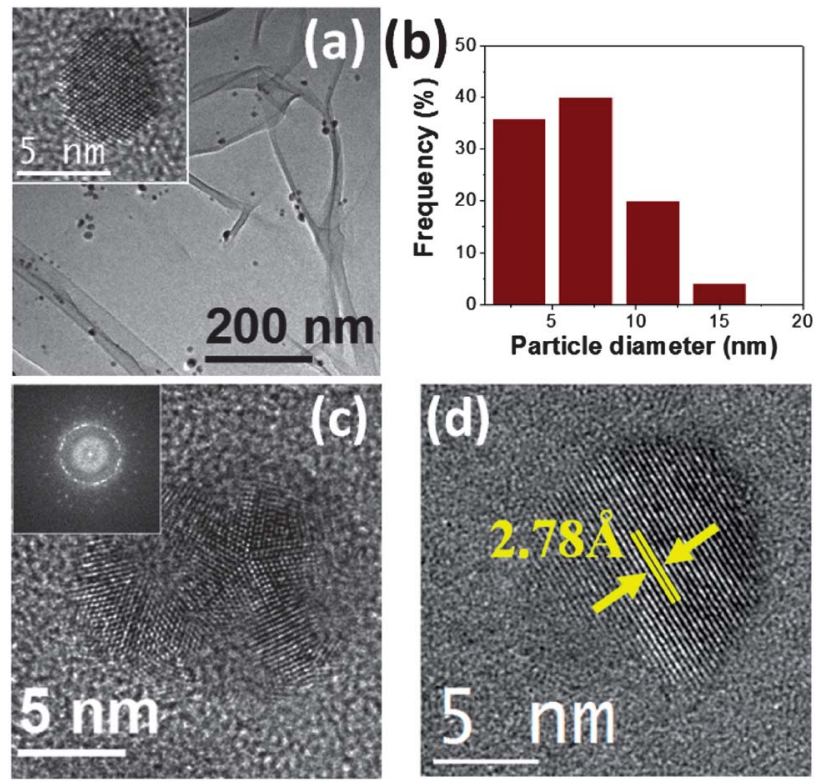

Fig. 2 (a) TEM micrograph of sample S-0.4\%. The inset is a HRTEM micrograph of an Ag single-crystal viewed along the [110] direction. (b) Particle size distribution of the sample S- $0.4 \%$. (c) HRTEM image of a twinned Ag nanoparticle of sample $\mathrm{S}-0.4 \%$. The inset is the corresponding FFT image. (d) HRTEM image of an isolated $\mathrm{C}_{14} \mathrm{H}_{14} \mathrm{AgN}_{3}$ nanodisk.

crystals that display a disk-like morphology with the $\langle 110\rangle$ direction perpendicular to their flat surface. Also, but in minor proportion, bigger silver fivefold twinned nanoparticles (Fig. 2c) and silver 4,4'-dimethyldiazoaminobenzene nanodisks with diameters around $12 \mathrm{~nm}$ (Fig. 2d) were observed.

Fig. 3a shows a TEM micrograph of the sample S- $42 \%$. This image reveals that the sample contains very fine particles with mean diameter of $4 \pm 2 \mathrm{~nm}$ (see their size distribution in Fig. 3b). In addition, a small percentage of bigger particles with sizes around $30 \mathrm{~nm}$, which tend to appear attached into clusters (highlighted by arrows in Fig. 3a), were also observed.

Fig. 3c shows a typical HRTEM image of an isolated nanoparticle with a diameter around $10 \mathrm{~nm}$ of sample S- $42 \%$. In this image, lattice fringes corresponding to the $\{111\}$ planes of fcc metallic silver are clearly observed. The corresponding FFT of the HRTEM, showed in Fig. 3d, displays spots that can be indexed to the [211] zone axis of the metallic silver. Interestingly, the majority of the examined particles of this sample were oriented along this zone axis, suggesting that these particles are rather flat falling the $\langle 211\rangle$ direction along the direction perpendicular to their flat surface, in contrast with the nanodisks of sample S- $0.4 \%$, whose $\langle 110\rangle$ direction is normal to the nanodisk plane. Moreover, additional spots associated to the $1 / 2(-13-1)$ and $1 / 2(-1-13)$ forbidden reflections are also found in the pattern presented in Fig. $3 \mathrm{~d}$. These results are significantly similar than those observed in Ag nanodisks prepared with red Capsicum annuum var. aviculare extracts, ${ }^{29}$ but different from the typical observations in plate-like nanostructures of $\mathrm{Ag}$ and $\mathrm{Au}$ prepared by conventional methods, in which the flat face is often parallel to $\{111\}$ and the forbidden $1 / 3\{224\}$
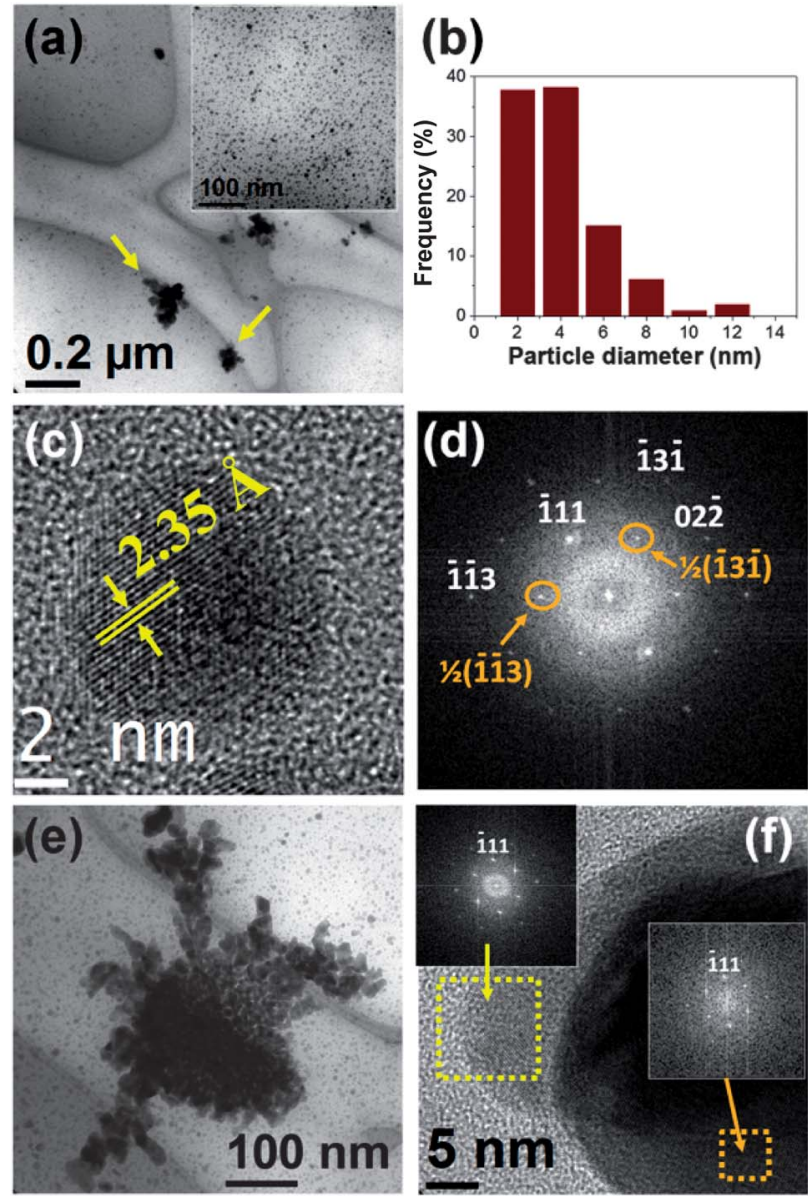

Fig. 3 (a) Low magnification TEM micrograph of sample S-42\%. (b) Histogram of size distribution of the finest particles of sample S-42\%. (c) HRTEM image of a nanodisk of sample S-42\%. The marked spacing of the fringes corresponds to the interplanar distance of $\{111\}$. (d) FFT of the image of panel (c). (e) TEM micrograph of a dendrite-like particle cluster. (f) HRTEM of two adjacent particles attached in the same particle cluster. Inset images are FFT patterns of the areas highlighted by squares.

reflections are usually observed in the SAED patterns recorded along the [111] zone axis. ${ }^{30,31}$

The appearance of forbidden reflections has been found in many metal fcc nanocrystalline structures with flattened morphologies. ${ }^{32,33}$ The occurrence of such reflections is usually attributed to the presence of different kinds of crystal defects, including the presence of parallel twining planes, ${ }^{34,35}$ dislocations, ${ }^{32}$ and (111) stacking faults. ${ }^{30}$ On the other hand, the observation of such reflections and the corresponding superlattice fringes in the HRTEM micrographs, also can be explained considering the low dimensionality of these materials and the physical principles on which the HRTEM and SAED measurements are based. ${ }^{33}$

Fig. 3e shows a TEM micrograph of an aggregate of particles of around $30 \mathrm{~nm}$ in size of sample S-42\%. In this particle cluster is remarkable the formation of "branches" that reflects the tendency of the bigger particles to self-assembly into dendriticlike structures. Moreover, HRTEM images of these supramolecular structures indicate that, usually, the attached particles into 
the clusters share the same crystallographic orientation. As an illustrative example, we present in Fig. 3f the HRTEM of two adjacent particles attached in the same particle cluster. Both particles have the same orientation and are viewed along the [211] direction. Moreover, it is worth noting that the FFT image corresponding to these particles (see the insets of Fig. 3f) exhibit the same features than those found for the smallest particles (see Fig. 2d), including the appearance of $1 / 2\{113\}$ forbidden spots.

Fig. 4a shows a TEM micrograph of the sample S-85\%. This image indicates that this sample presents several features similar than sample $\mathrm{S}-42 \%$. In this manner, this sample mostly consists of very fine nanoparticles with sizes around $4 \mathrm{~nm}$. Also, a population of particles with sizes around $50 \mathrm{~nm}$ that frequently appears forming aggregates (see the inset of Fig. 4a) is observed. In this case, the presence of these bigger particles is more notorious than in sample S- $42 \%$. SAED patterns of these bigger particles showed that they usually have a polycrystalline structure consistent with the fcc metallic silver, however additionally diffraction spots suggests the presence of silver 4,4'-dimethyldiazoaminobenzene (Fig. 4b). Fig. 4c presents a HRTEM image of one of such particles. In this image, $\mathrm{C}_{14} \mathrm{H}_{14} \mathrm{AgN}_{3}$ lattice fringes surrounding the $\mathrm{Ag}$ particle and with $d$-spacing of $2.78 \AA$ are clearly distinguished. These observations are very similar than those found in $\mathrm{Ag}$
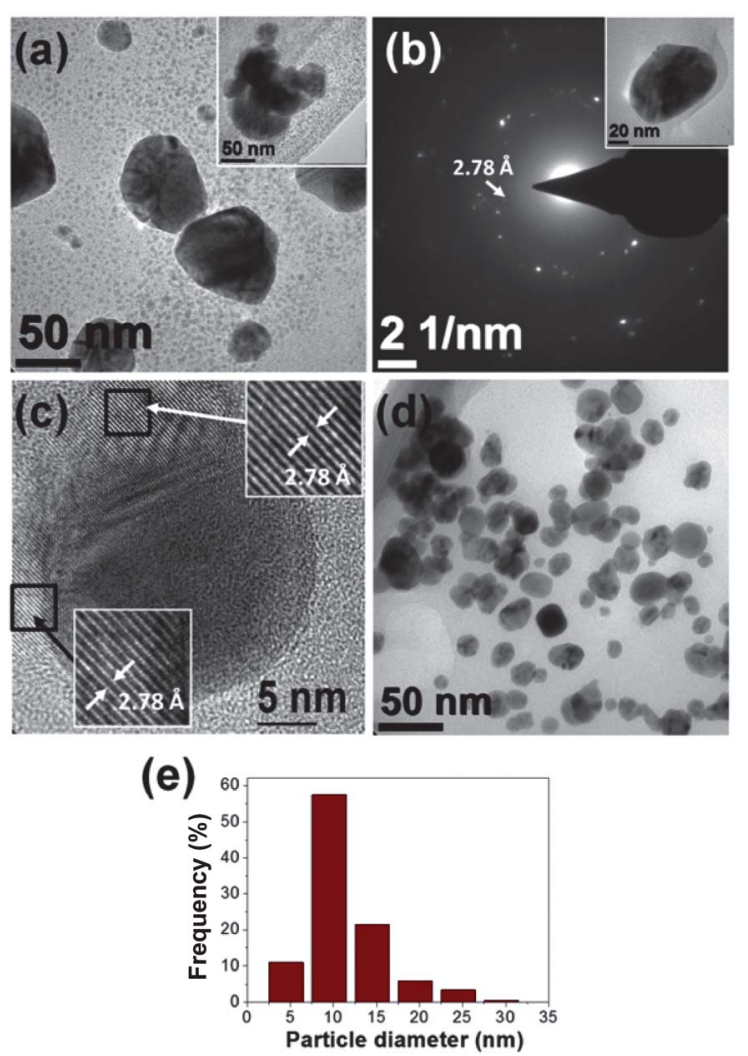

Fig. 4 (a) TEM micrographs of sample S-85\%. (b) SAED pattern corresponding to a single particle observed in the TEM micrograph of the inset image. (c) HRTEM micrograph of a single particle of sample S$85 \%$. (d and e) TEM micrograph and histogram of particle size distribution of sample S-85\% after two months of aging in solution and in environmental conditions, respectively. nanoparticles prepared with Capsicum annuum var. aviculare extracts, ${ }^{29}$ where it was found that $\mathrm{C}_{14} \mathrm{H}_{14} \mathrm{AgN}_{3}$ triazene nanodisks tend to be attached to $\mathrm{Ag}$ particle surface showing a capping agent like behaviour. Also, these results reveal that the crystallization of the organometallic disks is usual in the precipitation of $\mathrm{Ag}$-based particles from extracts of natural products.

In addition, dendritic structures of around $1.5 \mu \mathrm{m}$ in length and $1 \mu \mathrm{m}$ in width were also observed in sample S-85\% (see TEM images in Fig. 5a and b). These facts suggest that the attenuation of the stabilizing ability of the reaction medium via the reduction of the RJ concentration allows the formation of more amounts of bigger particles and the formation of dendritic structures by particle aggregation processes. EDS analysis confirmed that the principal component of the observed micrometric dendrite is $\mathrm{Ag}$ (Fig. 5g). The highmagnification TEM and HRTEM images (Fig. 5b and c) show that the branches of this dendrite are formed by coalesced monocrystalline particles that share the same crystallographic orientation and display a mesoporous structure with porous sizes of around $2-5 \mathrm{~nm}$ in diameter. This fact indicates that the initial polycrystalline particles experienced a structural reorganization during their self-assembly into the dendrite and the subsequent crystal growth by coalescence mechanisms.

Fig. 5c shows a HRTEM image of a branch of the dendritic structure. In this image, besides lattice fringes related to the face-centered cubic (fcc) phase of metallic silver, lattice fringes with $d$-spacing of around 2.46 ̊ are clearly distinguished. These
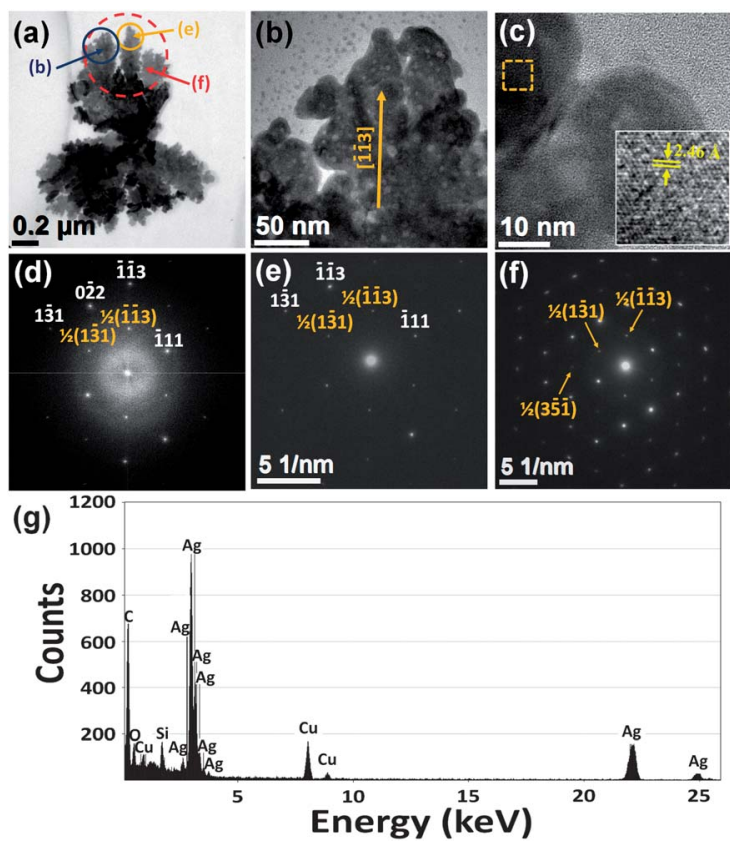

Fig. 5 ( $a$ and b) TEM micrographs of a dendrite observed in sample S$85 \%$. (c) HRTEM image of a dendrite "leaf". The inset is a magnification of the region marked by the discontinuous square. (d) FFT pattern corresponding to the image of panel (c). (e and f) SAED patterns of the areas highlighted by yellow and red squares, respectively, in panel (a). (g) EDS spectrum of the Ag dendrite of sample S-85\%. The different EDS peaks are labeled. 
fringes correspond to the $3 \times\{113\}$ superlattice spacing of the fcc metal silver crystal. The FFT image corresponding to the image of Fig. 5c shows a pattern of spots that can be indexed to the [211] zone axis of the metallic silver (see Fig. 5d), therefore, the flat surface of the dendrite is perpendicular to the [211] direction. Also, additional spots associated to the $3 \times\{113\}$ superlattice spacing are also found.

Another interesting feature of this dendritic structure is that the SAED patterns of different branches are very similar. In fact, several dendrite branches located at the top of Fig. 5a (marked with circles) share the same crystallographic orientation, as it is evidenced in the SAED patterns (see Fig. 5e and f). In addition, it is found that the axes of the branches are along the $\langle 113\rangle$ direction, therefore it is a preferential direction for the aggregation of the oriented attached nanocrystals.

From these experimental results we can conclude that, the formation of the dendrite started with the self-assembly of $\mathrm{Ag}$ nanoparticles. ${ }^{32}$ Afterwards, the intergrowth between nanocrystals probably occurs due to the tight packing of the nanoparticles into the dendritic structure. In addition, it is worth mentioning that close to dendrite branches appear very fine nanoparticles (see Fig. 5b). It indicates that the growth of the dendritic structure could continue by Ostwald ripening mechanism. ${ }^{36}$ To the best of our knowledge, this is the first time that the preparation of dendritic Ag structures at room temperature, mediated by natural derived products is reported. It could be useful to shed further light on the crystallization phenomena and the development of novel sensor technologies based on the excellent SERS properties of Ag dendritic structures. ${ }^{37}$

The TEM examination of the sample S-85\% after two months of aging in environmental conditions revealed that the small particles considerably grew with an extension of the mean diameter up to $11 \pm 4 \mathrm{~nm}$ (see Fig. $4 \mathrm{~d}$ and e). On the other hand, micrometric dendrites were not found, suggesting the instability of such structures.

In order to gain further information about the thermal stability of the crystalline phases of metallic silver and silver $4,4^{\prime}$-dimethyldiazoaminobenzene, the sample S-0.4\% was thermally treated as it is described in the Methods section. Fig. 1Sa $\dagger$ displays the XRD pattern of the resulting powder (i.e. sample S$0.4 \% \mathrm{~S}$ ). It is observed that the diffraction peaks ascribed to the silver $4,4^{\prime}$-dimethyldiazoaminobenzene phase are considerably more intense with respect to the peaks of the metallic Ag phase, and their broadening significantly decreased with respect to the corresponding peaks of sample $\mathrm{S}-0.4 \%$. It indicates that the crystallization of the $\mathrm{C}_{14} \mathrm{H}_{14} \mathrm{AgN}_{3}$ phase is favored with the thermal treatment. Fig. 1Sb $\dagger$ displays a TEM micrograph of this sample where nanoparticles of diameters around $4 \mathrm{~nm}$ embedded into a matrix are observed. Fig. 1Sc $\dagger$ presents a HRTEM micrograph where lattice fringes corresponding to fcc metallic silver and silver 4,4'-dimethyldiazoaminobenzene are observed (see also the corresponding FFT image in Fig. $1 \mathrm{Sd} \dagger$ ).

\section{Studies by FTIR, Raman and UV-visible spectroscopies}

In contrast with the initial light yellow solution, as-prepared samples exhibited a yellowish brown color, being samples

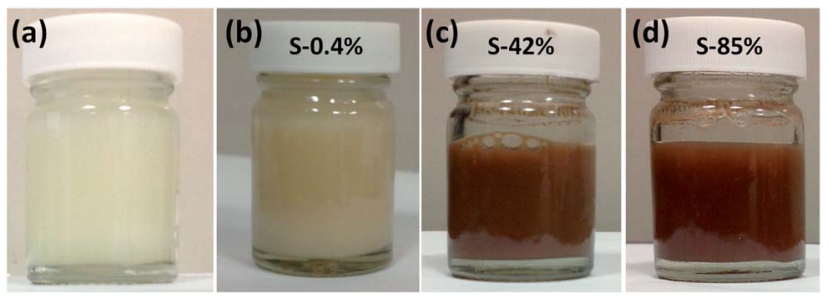

Fig. 6 Photographs of (a) initial solution (experiment S-85\%) before reactions and solutions after reactions of experiments (b) S- $0.4 \%$, (c) S$42 \%$ and (d) S- $85 \%$.

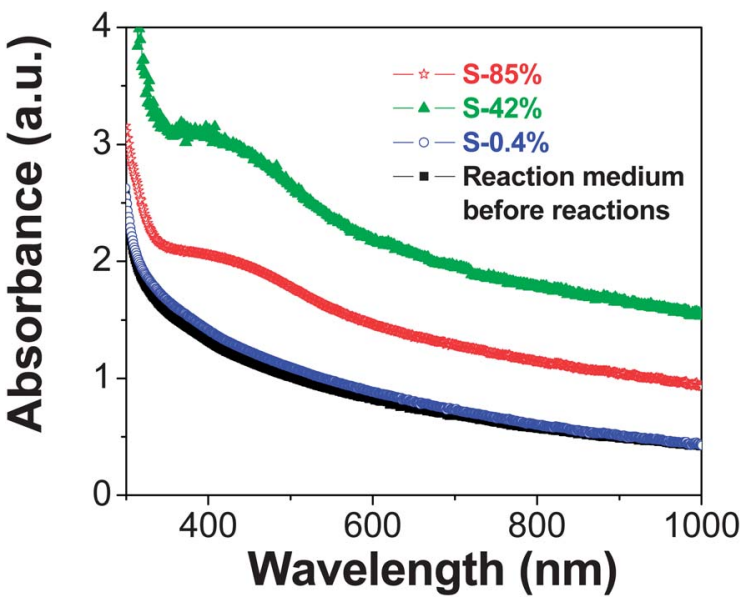

Fig. 7 UV-Visible spectra of the initial solution (experiment S-85\%) before reactions and solutions after reactions (experiments S- $0.4 \%$, S$42 \%$ and $\mathrm{S}-85 \%$ ).

S-42\% and S-85\% considerably darker than sample S- $0.4 \%$ (see Fig. 6).

Fig. 7 shows UV-visible spectra of the samples S-0.4\%, S-42\% and $\mathrm{S}-85 \%$ after reactions compared with a typical spectrum recorded for an initial solution before reactions. The spectra of samples $\mathrm{S}-42 \%$ and $\mathrm{S}-85 \%$ clearly present a surface plasmon resonance (SPR) extinction band with an absorbance maximum at around $430 \mathrm{~nm}$, confirming the presence of fine $\mathrm{Ag}$ particles. In the case of sample S- $0.4 \%$, a SPR band is not distinguished, probably due to the low particle concentration of this sample.

FTIR and Raman spectroscopy analyses were carried out to gain information about the formation mechanism of silverbased nanostructures in RJ solutions. RJ is very rich in reducing compounds, mainly proteins (about $50 \%$ of the dry mass) and carbohydrates (about $30 \%$ of the dry mass). ${ }^{38}$

Fig. 8 shows the FTIR spectra recorded from dried royal jelly and the silver-based samples. The spectra of the Ag-based samples display well-defined characteristic bands observed at around 3300, 3070, 2950, 1670, 1525, 1448, 1400, and $1058 \mathrm{~cm}^{-1}$ that appear also in the spectrum of the royal jelly but slightly shifted and/or with difference relative intensity. Such bands can be mainly associated to residues of proteins and carbohydrates of RJ. This fact suggests that biomolecules of RJ are conjugated with the Ag-based nanoparticles. In this manner, the broad band observed at the wavenumber range of $3500-2750 \mathrm{~cm}^{-1}$ 


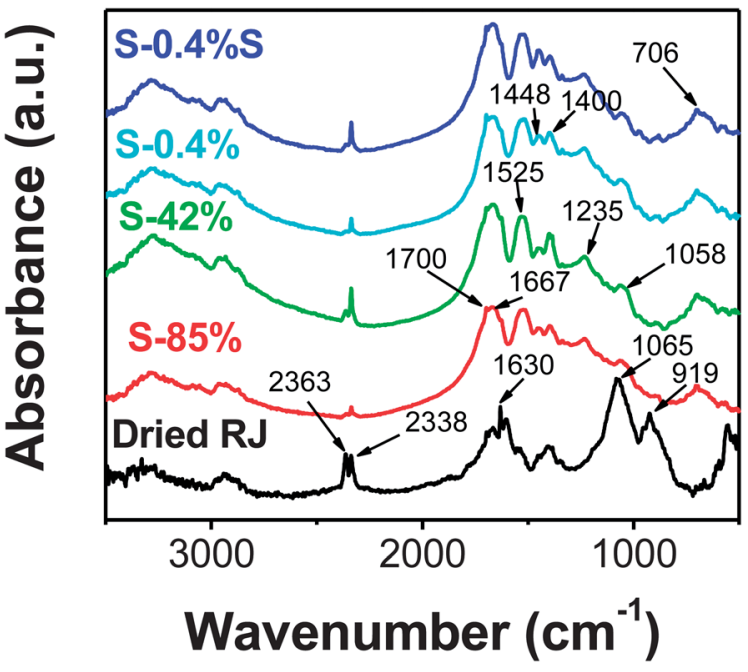

Fig. 8 FTIR spectra of dried royal jelly and the Ag-based samples.

and with three maximums around 3300,3070 and $2950 \mathrm{~cm}^{-1}$ is associated to contributions of the $\mathrm{N}-\mathrm{H}, \mathrm{O}-\mathrm{H}$ and $\mathrm{C}-\mathrm{H}$ stretching vibrations. The bands at around 1670 and $1525 \mathrm{~cm}^{-1}$ corresponds to the $\mathrm{C}=\mathrm{O}$ stretching vibration of peptide bond (amide I band) and the $\mathrm{N}-\mathrm{H}$ bending vibration (amide II band) of proteins, respectively. ${ }^{39}$ Also, the $\mathrm{N}=\mathrm{N}$ vibration of the silver triazene ${ }^{40}$ could contribute to the band around $1525 \mathrm{~cm}^{-1}$ of the spectra of the Ag-based samples. The peaks at 1448 and $1400 \mathrm{~cm}^{-1}$ could correspond to $\mathrm{C}-\mathrm{OH}$ groups. The relative intensity of these peaks is different in the Ag-based samples and the band at $1448 \mathrm{~cm}^{-1}$ is significantly more marked for sample S- $0.4 \% \mathrm{~S}$. It is probably due to the additional contribution of the $\mathrm{N}=\mathrm{N}$ vibrations of the triazenic compound present in the $\mathrm{Ag}$-based samples. Interestingly, the intense peak found at $1065 \mathrm{~cm}^{-1}$ in the RJ spectrum, which can be ascribed to $-\mathrm{C}-\mathrm{O}$ vibration of carbohydrates, appears strongly diminished and shifted to $1058 \mathrm{~cm}^{-1}$ in the Ag-based samples. The band at $1235 \mathrm{~cm}^{-1}$ observed in the spectra of the Ag-based samples could be associated to stretching vibrations of $\mathrm{C}-\mathrm{O}$ in carbohydrates and the $\mathrm{C}-\mathrm{N}$ stretching and $\mathrm{N}-\mathrm{H}$ bending vibrations (amide III band) of proteins.

From these results, we can conclude that RJ proteins and carbohydrates (reducing sugars) are the responsible of the bioreduction of the $\mathrm{Ag}^{+}$ions, and as a consequence, residues of aminoacids and oxidized sugars are bounded to the Ag nanodisk surface. This hypothesis is in agreement with the tendency of proteins to bind to Ag surfaces through the amine and/or the carboxylate ion groups found in other works. ${ }^{7,41}$ On the other hand, the oxidation of carbohydrates during the bioreduction should produce carboxylic acids bounded to the particle surface, and it is evidenced with the observation of FTIR bands in the spectra of the nanoparticles at around 2900-3000, 1720$1650,1400,1230$ and $930 \mathrm{~cm}^{-1}$ ascribed to the $\nu(\mathrm{O}-\mathrm{H}), \nu(\mathrm{C}=\mathrm{O})$, $\delta(\mathrm{C}-\mathrm{O}-\mathrm{H}), \nu(\mathrm{C}-\mathrm{O})$ and $\mathrm{O}-\mathrm{H}$ out-of-plane bend vibration modes, respectively.

Fig. 9 shows the Raman spectra of RJ and the biosynthesized samples. The spectra of the Ag-based samples exhibit broad and

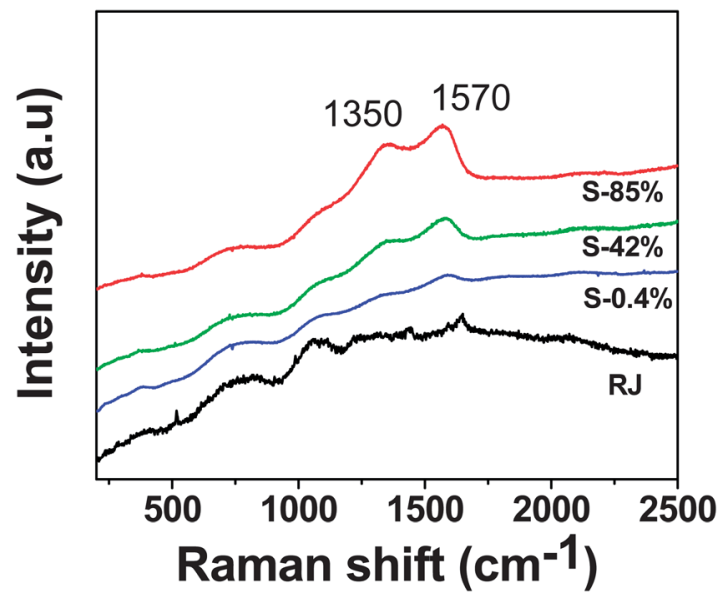

Fig. 9 Raman spectra of aqueous solution of royal jelly and water suspensions of the Ag-based nanostructures synthesized using royal jelly.

intense bands at 1350 and $1570 \mathrm{~cm}^{-1}$ that correspond to symmetric and asymmetric $\mathrm{C}=\mathrm{O}$ stretching vibrations of the carboxylate ions. ${ }^{42-44}$ The enhancement of these bands confirms that carboxylate ions are directly bound to $\mathrm{Ag}$ nanoparticle surface falling the $\mathrm{C}=\mathrm{O}$ groups perpendicular to the particle surface. ${ }^{42,45-47}$

\section{Antibacterial assays}

Fig. 10 and 11 depict the results of the antibacterial assays carried out with as-prepared samples S- $42 \%$ and S- $85 \%$. Both samples exhibited significant antibacterial activity against Gram-negative bacteria (S. typhimurium) and Gram-positive bacteria (S. aureus). In this manner, clear non-growth halos were observed in the agar experiments (Fig. 10), where the halo diameters of the experiments with sample S- $85 \%$ were larger than those of sample S- $42 \%$. On the other hand, the MIC values obtained for experiments with $S$. aureus (around 8 and $4 \mu \mathrm{g} \mathrm{ml}^{-1}$ in $\mathrm{Ag}$ content, respectively) and S. typhimurium (around $4 \mu \mathrm{g}$ $\mathrm{ml}^{-1}$ in $\mathrm{Ag}$ content for both samples), estimated as the concentration for which the absorbance at $\lambda=540 \mathrm{~nm}$ experienced a strong fall (Fig. 11), were of the same order than the lowest MIC values reported using Ag nanoparticles. ${ }^{48,49}$

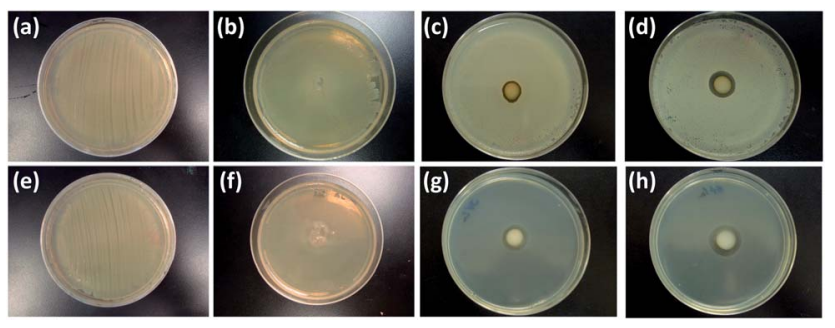

Fig. 10 Bacteria grown on agar plates. (a-d) Results of the experiments with S. aureus: (a) control experiment, (b) in presence of RJ, (c) in presence of sample S- $42 \%$ and (d) in presence of sample S-85\%. (eh) Results of the experiments with $S$. typhimurium: (e) control experiment, (f) in presence of $\mathrm{RJ},(\mathrm{g})$ in presence of sample $\mathrm{S}-42 \%$ and $(\mathrm{h})$ in presence of sample S-85\%. 

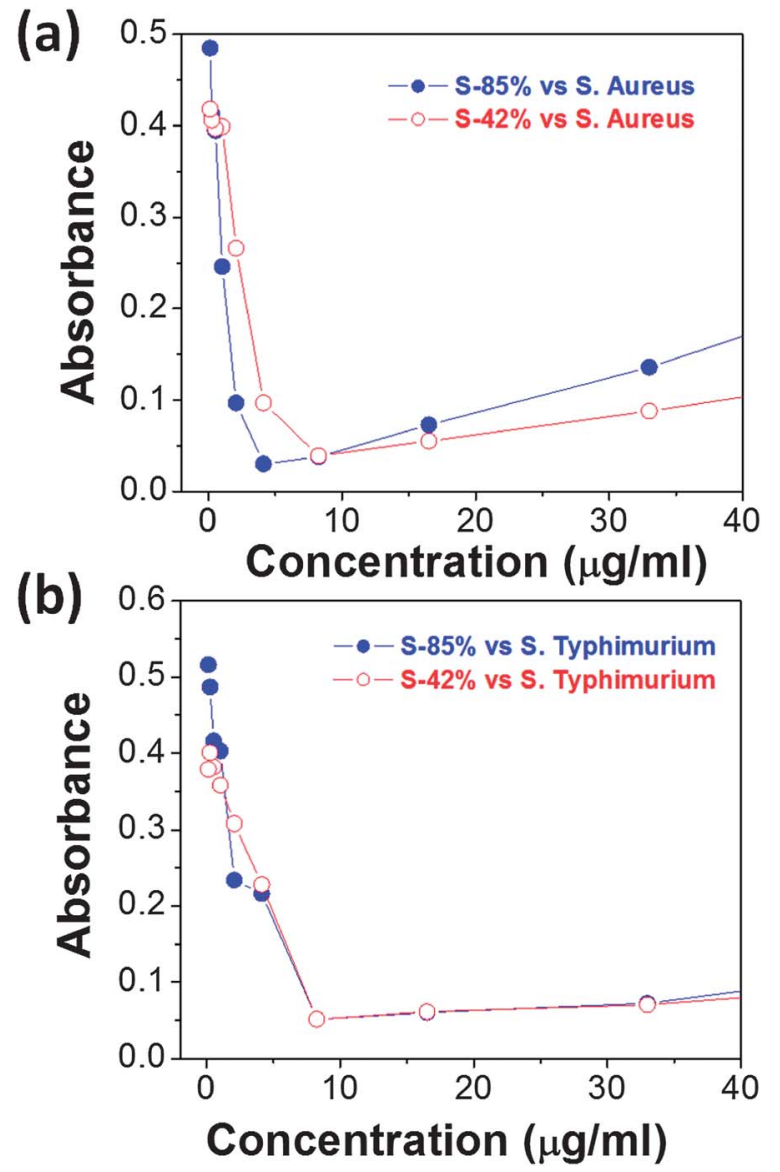

Fig. 11 Absorbance at $\lambda=540 \mathrm{~nm}$ (b) of (a) $S$. aureus and (b) $S$. typhimurium as a function of the concentration of samples $\mathrm{S}-42 \%$ and S- $85 \%$.

Therefore, the Ag-based nanoparticles synthesized using RJ have a very promising potential as antibacterial agents.

\section{Conclusions}

It has been demonstrated for the first time that Ag-based nanostructures can be synthesized at room temperature using aqueous royal jelly solutions as reducing agent of $\mathrm{Ag}^{+}$ions. The obtained product mainly consisted of ultra-fine Ag singlecrystals with disk-like morphologies and diameters of around 4 $\mathrm{nm}$. In addition, nanodisks of silver $4,4^{\prime}$-dimethyldiazoaminobenzene with diameters around $10 \mathrm{~nm}$ and twined fine $\mathrm{Ag}$ particles have been found. The growth of these nanostructures and their microstructural characteristics were controlled with the $\mathrm{AgNO}_{3} / \mathrm{RJ}$ weight ratio fixed during the synthesis. In such manner, when this ratio was increased from $0.42 \%$ up to $42 \%$ or $85 \%$, the perpendicular direction to the $\mathrm{Ag}$ nanodisk plane changed from the $\langle 110\rangle$ to $\langle 211\rangle$ direction. Also, the metallic silver and the organometallic nanodisks tended to growth by aggregation and coalescence to form particles of several tens of $\mathrm{nm}$ that, in turn, self-assemble into Ag dendritic supramolecular structures. Antibacterial assays indicated that these nanostructures prepared in presence of RJ exhibit important antibacterial activity against Gram-positive and Gram-negative bacteria.

\section{Acknowledgements}

We gratefully acknowledge support from the "Secretaría de Educación Publica (SEP-PROMEP)" under its program named "Fortalecimiento de Cuerpos Académicos", reference UANL-CA305, and by the Mexican Council of Science and Technology (CONACYT) and Universidad Autónoma de Nuevo León (UANL) under research projects CB-179486 and PAICYT-CE793-11, respectively.

\section{References}

1 H. H. Lara, E. N. Garza-Treviño, L. Ixtepan-Turrent and D. K. Singh, J. Nanobiotechnol., 2011, 9, 30.

2 H. Tan, R. Santbergen, A. H. M. Smets and M. Zeman, Nano Lett., 2012, 12, 4070.

3 K. Kneipp, Y. Wang, H. Kneipp, L. T. Perelman, I. Itzkan, R. R. Dasari and M. S. Feld, Phys. Rev. Lett., 1997, 78, 1667.

4 I. Hussain, M. Brust, A. J. Papworth and A. I. Cooper, Langmuir, 2003, 19, 4831.

5 J.-H. Kim, W. W. Bryan and T. R. Lee, Langmuir, 2008, 24, 11147.

6 A. Annamalai, V. L. P. Christina, D. Sudha, M. Kalpana and P. T. V. Lakshmi, Colloids Surf., B, 2013, 108, 60.

7 D. Philip, Spectrochim. Acta, Part A, 2010, 75, 1078.

8 D. Philip, Spectrochim. Acta, Part A, 2009, 73, 650.

9 D. S. Shenya, J. Mathewa and D. Philip, Spectrochim. Acta, Part A, 2011, 79, 254.

10 M. Ganeshkumar, M. Sathishkumar, T. Ponrasu, M. Girija Dinesh and L. Suguna, Colloids Surf., B, 2013, 106, 208.

11 V. Snitka, D. O. Naumenko, L. Ramanauskaite, S. A. Kravchenko and B. A. Snopok, J. Colloid Interface Sci., 2012, 386(1), 99.

12 G. V. White II, P. Kerscher, R. M. Brown, J. D. Morella, W. McAllister, D. Dean and C. L. Kitchens, J. Nanomater., 2012, 2012, 730746.

13 S. M. Reddy, K. K. R. Datta, C. S. M. Eswaramoorthy and B. V. S. Reddy, Nanosci. Nanotechnol. Lett., 2012, 4, 420.

14 R. Venu, T. S. Ramulu, S. Anandakumar, V. S. Rani and C. G. Kim, Colloids Surf., A, 2011, 384, 733.

15 Z. Kerem, D. Chetrit, O. Shoseyov and G. Regev-Shoshani, J. Agric. Food Chem., 2006, 54(26), 10288.

16 N. Almaraz, M. G. Campos, J. A. Avila, N. Naranjo, J. Herrera and L. S. Gonzalez, J. Food Compos. Anal., 2007, 20(2), 119.

17 A. Kosinska, M. Karamac, K. Penkacik, A. Urbalewicz and R. Amarowicz, Eur. Food Res. Technol., 2011, 233, 213.

18 T. Nagai, M. Sakai, R. Inoue, H. Inoue and N. Suzuki, Food Chem., 2001, 75, 237.

19 A. G. P. Samaranayaka and E. C. Y. Li-Chan, J. Funct. Foods, 2011, 3, 229.

20 F. Shahidi and Y. Zhong, J. Agric. Food Chem., 2011, 59, 3499. 21 S. Buratti, S. Benedetti and M. S. Cosio, Talanta, 2007, 71, 1387.

22 A. von Planta, Z. Physiol. Chem., 1888, 12, 327. 
23 C. C. Lucas, Can. Med. Assoc. J., 1942, 406.

24 B. Han, C. Li, L. Zhang, Y. Fang, M. Feng and J. Li, J. Agric. Food Chem., 2011, 59(18), 10346.

25 N. Weaver, N. C. Johnston, R. Benjamis and J. H. Law, Lipids, 1968, 3, 535.

26 M. F. Ramadan and A. Al-Ghamdi, J. Funct. Foods, 2012, 4, 39.

27 B. D. Cullity and S. R. Stock, Elements of X-ray Diffraction, Prentice-Hall, Englewood Cliffs, NJ, 2001.

28 J. M. Zuo and J. C. Mabon, Web-based Electron Microscopy Application Software: Web-EMAPS, Microsc. Microanal. 10 (suppl. 2), 2004, http://emaps.mrl.uiuc.edu/.

29 R. Mendoza-Reséndez, N. O. Nuñez, E. D. Barriga-Castro and C. Luna, $R S C A d v .$, 2013, 3, 20765.

30 J. Li, D. Ingert, Z. L. Wang and M. P. Pileni, J. Phys. Chem. B, 2003, $107(34), 8717$.

31 M. Yamamoto, Y. Kashiwagi, T. Sakata, H. Mori and M. Nakamoto, Chem. Mater., 2005, $17(22), 53091$.

32 L. Lu, A. Kobayashi, Y. Kikkawa, K. Tawa and Y. Ozaki, J. Phys. Chem. B, 2006, 110, 23234.

33 J. Reyes-Gasga, A. Gómez-Rodríguez, X. Gao and M. JoséYacamán, Ultramicroscopy, 2008, 108, 929.

34 D. Cherns, Philos. Mag., 1974, 30, 549.

35 D. W. Pashley and M. J. Stowell, Philos. Mag., 1963, 8, 127.

36 B. Jia and L. Gao, Cryst. Growth Des., 2008, 8, 1372.

37 R. Xiong, C. Lu, W. Zhang, Z. Zhou and X. Zhang, Carbohydr. Polym., 2013, 95, 214.

38 L. I. Barnutiu, L. A. Marghitas, D. S. Dezmirean, C. M. Mihai and O. Bobis, J. Anim. Sci. Biotechnol., 2011, 44(2), 67.
39 J. Coates, Interpretation of Infrared Spectra, A Practical Approach, in Encyclopedia of Analytical Chemistry, ed. R. A. Meyers, John Wiley \& Sons Ltd, Chichester, 2000.

40 F. Zimmermann, T. H. Lippert, C. H. Beyer, J. Stebani, O. Nuyken and A. Wokaun, Appl. Spectrosc., 1993, 47, 986.

41 A. Ahmad, S. Senapati, M. I. Khan, R. Kumar and M. Sastry, Langmuir, 2003, 19, 3550.

42 A. J. Kora, R. B. Sashidhar and J. Arunachalam, Process Biochem., 2012, 47, 1516.

43 M. Baia, S. Astilean and T. Iliescu, Raman and SERS Investigations of Pharmaceuticals, Springer-Verlag, Berlin Heidelberg, 2008.

44 D. W. Mayo, F. A. Miller and R. W. Hannah, Course Notes on the Interpretation of Infrared and Raman Spectra, John Wiley \& Sons, Inc., Hoboken, New Jersey, 2003.

45 E. Podstawka, Y. Ozaki and L. M. Proniewicz, Appl. Spectrosc., 2004, 58(10), 1147.

46 E. Podstawka, Y. Ozaki and L. M. Proniewicz, Appl. Spectrosc., 2004, 58(5), 570.

47 P. R. Selvakannan, R. Ramanathan, B. J. Plowman, Y. M. Sabri, H. K. Daima, A. P. O'Mullane, V. Bansal and S. K. Bhargava, Phys. Chem. Chem. Phys., 2013, 15, 12920.

48 W. R. Li, X. B. Xie, Q. S. Shi, H. Y. Zeng, Y. S. Ou-Yang and Y. B. Chen, Appl. Microbiol. Biotechnol., 2010, 85, 1115.

49 A. Panáček, L. Kvítek, R. Prucek, M. Kolář, R. Večeřová, N. Pizúrová, V. K. Sharma, T. Nevěčná and R. Zbořil, J. Phys. Chem. B, 2006, 110, 16248. 